\title{
STATIC FINITE-ENERGY SOLUTIONS OF GAUGE FIELDS WITH SEPARATED RADIAL VARIABLE
}

\author{
L. MICHEL 1 \\ Physics Department, University of Michigan, Ann Arbor, Michigan 48104, USA \\ L. O'RAIFEARTAIGH \\ DIAS, Dublin 4, Ireland \\ and \\ Kameshwar C. WALI ${ }^{2}$ \\ Physics Department, Syracuse University, Syracuse, New York 13210, USA
}

Received 10 February 1977

\begin{abstract}
For arbitrary compact gauge group $\mathrm{G}$ and real representations of the Higgs fields, we seek static finite-energy solutions for which the radial dependence of the fields is factorized. We find that the gauge fields vanish outside a fixed $S O$ (3) subgroup of $G$, and that inside $S O(3)$ they reduce to the 't Hooft-Polyakov solution with unit magnetic charge. The Higgs fields may belong to any integer representation of this $\mathrm{SO}(3)$.
\end{abstract}

Let $\mathrm{G}$ be a compact Lie group and

$\mathcal{L}=-\frac{1}{4}\left(F_{\mu \nu}, F_{\mu \nu}\right)-\frac{1}{2}\left(D_{\mu} \Phi, D_{\mu} \Phi\right)-V(\Phi)$,

a Yang-Mills-Higgs Lagrangian density for $\mathrm{G}$, where $(A, B)$ denotes inner product in $\mathrm{G}$-spaces, $\Phi$ belongs to any real representation $T(g)$ of $\mathrm{G}$, and

$D_{\mu} \Phi^{A}=\nabla_{\mu} \Phi^{A}+e A_{\mu}^{\alpha} t_{A B}^{\alpha} \Phi^{B}$

$D_{\mu} A_{\nu}^{\alpha}=\nabla_{\mu} A_{\nu}^{\alpha}+e c_{\beta \gamma}^{\alpha} A_{\mu}^{\beta} A_{\nu}^{\gamma}$

$F_{\mu \nu}=\frac{1}{e}\left[D_{\mu}, D_{\nu}\right]=\nabla_{\mu} A_{\sim}-\nabla_{\nu} \sim_{\mu}+e\left[{\underset{\sim}{\mu}}_{\mu},{\underset{\sim}{A}}_{\nu}\right]$,

$\stackrel{A}{\sim}_{\mu}=A_{\mu}^{\alpha} \tau^{\alpha}$,

where $c_{\beta \gamma}^{\alpha}$ are the structure constants of $\mathrm{G}$ and $\tau^{\alpha}$ and $t^{\alpha}$ are the (real anti-symmetric) generators of the adjoint $\tau(g)$ and $T(g)$ representations of $\mathrm{G}$ respectively. Classically there is no restriction on $V(\Phi)$ except that it be G-invariant and bounded below, but for QFT renormalizability requires that it be a polynomial of fourth degree. Furthermore for the occurrence of

\footnotetext{
1 Permanent address: IHES, Bures-sur-Yvette, 91440, France

${ }^{2}$ Work supported in part by the U.S. Energy Research and Development Administration, contract No. EY-76-S-02:3533.
}

Higgs mechanism, the second degree term in $V(\Phi)$ must have a negative coefficient $\$ \mathbf{1}$. When the fields are static (and $A_{0}$ is zero), the Hamiltonian and field equations corresponding to $\mathcal{H}$ reduce to

$$
\begin{aligned}
\mathcal{T C} & =\int \mathcal{C}(x) \mathrm{d}^{3} x \\
& =\int\left\{\frac{1}{2}(\boldsymbol{E}, \boldsymbol{E})+\frac{1}{2}(\boldsymbol{D} \Phi, \boldsymbol{D} \Phi)+V(\Phi)\right\} \mathrm{d}^{3} x
\end{aligned}
$$

and

$D^{2} \Phi=\frac{\partial V}{\partial \Phi}, \quad \underline{D} \times F^{\alpha}=-e\left(\Phi, t^{\alpha} D^{\Phi}\right)$,

respectively. The standard boundary conditions for finite-energy solutions [2] ${ }^{\ddagger 2}$ of (3), (4) are that $A(x)$ and $\Phi(x)$ bc rcgular everywhere, including the origin, and that

${ }^{\ddagger 1}$ We choose this coefficient to be $-\mu^{2} / 2$. For a general review of gauge theories and spontaneous symmetry breaking, see e.g. [1].

\$2. Here we mean solutions throughout all three-space. Generalizations of ref. [2], using topological considerations on the sphere at infinity have been considered elsewhere. For a partial list, containing other references, see [3]. 
$\lim _{r \rightarrow \infty} \operatorname{Re} \underset{\sim}{A}(r, \omega)=\underset{\sim}{a}(\omega)$ and $\lim _{r \rightarrow \infty} \Phi(r, \omega)-c \phi(\omega)$

$(\phi(\omega), \phi(\omega))=1, \quad \omega=(\theta, \phi)$,

where $\phi(\omega)$ lie on a group orbit determined by the form of the Higgs potential, $c$ is a non-zero constant, and $\underset{\sim}{\boldsymbol{a}}(\omega)$ and $\phi(\omega)$ are understood to be finite. The convergence of the kinetic term for $\Phi$ in (4) requires also that

$\underset{\sim}{d} \phi(\omega)=0$, where $\underset{\sim}{d}=\partial+a^{\alpha} t^{\alpha}, \partial=r \nabla$.

Eq. (7) states that the $\phi(\omega)$ for different $\omega$ are related by a group transformation, that is $\phi(\omega)$ can be written in the form $\phi(\omega)=u(g(\omega)) \phi(0)$, where $g(\omega) \in \mathrm{G}$. Eq. (7) can also be written as

$\boldsymbol{J} \phi(\omega)=0$, where $\boldsymbol{J}=-\hat{\boldsymbol{r}} \times \underset{\sim}{\boldsymbol{d}}=\boldsymbol{L}+\boldsymbol{b}^{\alpha}(\omega) t^{\alpha}$,

where $\boldsymbol{J}$ is the covariant angular momentum.

It is useful to digress a little at this point, to study the integrability condition for (8). One sees by inspection that it is

$\underset{\sim}{f} \phi(\omega)=0$, where $f_{i}=\epsilon_{i j k}\left\{\left[J_{i}, J_{j}\right]-\epsilon_{i j k} J_{k}\right\}$,

and a straightforward computation shows that

$\underset{\sim}{f}=\underset{\sim}{\hat{r} f}, \quad \underset{\sim}{f}=\lim _{r \rightarrow \infty} r^{2}(\hat{r} \cdot F)=\underset{\sim}{2 u}+\underset{\sim}{v}$,

where

$2 \underset{\sim}{u}=-\boldsymbol{L} \cdot \underset{\sim}{a}$ and $\underset{\sim}{v}=-\epsilon_{i j k} \hat{r}_{i \sim} a_{j} a_{k}$

Thus $f$ is purely radial. The scalar field $f$ is the analogue, on the sphere, of the field $\underset{\sim}{\underset{F}{*}}$ in Minkowski space. It measures (from (9)) the failure of the generators of the relevant geometrical group to close under commutation, and if it vanishes, the relevant gauge field (the transverse part of $a(\omega)$ ) can be gauged to zero. Since $f$ is a scalar in ordinary space, a vector in the adjoint representation of $\mathrm{G}$, and is in the little group of $\phi(\omega)$ from (9), it is a natural candidate for defining the electromagnetic direction in the adjoint representation of $\mathrm{G}$.

It is also useful to note that the Higgs mechanism equips the gauge fields with a mass-matrix $e^{2} M_{\alpha \beta}$ in the usual way, where

$M_{\alpha \beta}=c^{2}\left(t^{\alpha} \phi, t^{\beta} \phi\right)$.
The mass-matrix $M_{\alpha \beta}$ appears to depend on $\omega$, but the physical masses are $\omega$-independent because, as can be shown at once from (7), the covariant derivative of $M_{\alpha \beta}$ vanishes, i.c.,

$\mathrm{d}_{i} M_{\alpha \beta} \equiv \partial_{i} M_{\alpha \beta}+a_{i}^{\gamma}\left\{c_{\alpha \alpha^{\prime}}^{\gamma} M_{\alpha^{\prime} \beta}+c_{\beta \beta^{\prime}}^{\gamma} M_{\alpha \beta^{\prime}}\right\}=0$.

From (7) it also follows that

$M_{\alpha \beta} a_{i}^{\beta}=c^{2}\left(\phi, t^{\alpha} \partial_{i} \phi\right)$

which expresses the massive gauge-fields in terms of the Higgs currents.

After this digression, which is completely general, since it contains no assumption of variable separation, we now turn to our main consideration which is to seek radially separated solutions of the Hamiltonian system (4), (5). That is, we seek solutions of the form
(a) $\Phi^{A}(r, \omega)=\phi^{A}(\omega)\left(\frac{S(r)}{r}\right)$
(b) $\quad e A_{i}^{\alpha}(r, \omega)=a_{i}^{\alpha}(\omega)\left(\frac{R(r)}{r}\right)$.

We assume that the separation (15) takes place in the Landau gauge, in which $\operatorname{div} \underset{\sim}{A}$ is zero, and we note that (15a) is then a condition on $\Phi$, not a choice of gauge. Eq. (15) is a natural generalization of the 't Hooft-Polyakov [2] ansatz, but here no a priori assumption is made about the group $\mathrm{G}$ or about the form of $\phi(\omega)$ and $\underset{\sim}{a}(\omega)$. In particular $\Phi(\omega)$ and $\underset{\sim}{a}(\omega)$ are not assumed to be spherically symmetric [4].

The radial separation (15) actually reduces the Landau gauge condition to two separate conditions, namely,

$\partial \cdot \underset{\sim}{a}(\omega)=0$ and $\hat{r} \cdot \underset{\sim}{a}(\omega)=0$.

The program now is to insert (15) and (16) in the equations of motion (5), to obtain separated equations for the angular and radial functions, and then to solve the separated equations (or at least the angular ones). Making the insertion we find the angular equations are
(a) $\dot{\partial}^{2} \underset{\sim}{a}=-L(L+1) \underset{\sim}{a}$,
(b) $\partial^{2} \phi=-l(l+1) \phi$,
(c) $\left({\underset{\sim}{a}}_{i}, \underset{i}{a}\right)=v^{2}$,
(d) $\left[{\underset{\sim}{a}}_{i}\left[{\underset{\sim}{a}}_{i}, \underset{\sim}{a} j\right]\right]=-\frac{\nu^{2}}{N} \underset{\sim}{a} j$,
(e) $a_{i}^{\alpha}=\frac{1}{n}\left(\phi, t^{\alpha} \partial_{i} \phi\right)$, (f) $\mathrm{d}_{i} f=0$, 
where $L, l, \nu, n$ and $N$ are positive constants with $l(l+1)=n \nu^{2}$. The corresponding radial equations are

$r^{2} S^{\prime \prime}=S\left[l(l+1)(1-R)^{2}+\mu^{2}(S-r)(\sigma S+r)\right]$,

$r^{2} R^{\prime \prime}=(1-R)\left[L(L+1) R(1-\epsilon R)-e^{2} n c^{2} S^{2}\right]$,

where

$\epsilon=l(l+1) / L(L=1) N n$,

and the dimensionless positive constant $\sigma$ depends on the precise form of the potential $V(\Phi)$. For example, when

$V(\Phi)=\frac{\lambda}{4}(\Phi, \Phi)^{2}-\frac{\mu^{2}}{2}(\Phi, \Phi)$, then $\sigma=1$ and

$c^{2}=\mu^{2} / \lambda$.

In general if there are no third degree invariants in $V(\Phi)$ we have $\sigma=1$. Before proceeding to solve the angular equations, it is perhaps worthwhile to analyze them briefly. The first two equations in (17) show that $\underset{\sim}{\boldsymbol{a}}(\omega)$ and $\phi(\omega)$ are spherical harmonics of definite order (and that $L$ and $l$ are positive integers). Next, from the definition (11) of $y(\omega)$ and (17d) one sees that $\underset{\sim}{v}(\omega)$ and the two transverse components of $\underset{\sim}{\boldsymbol{a}}(\omega)$ form an $S U(2)_{\omega}$ Lie subalgebra of the Lie algebra $\mathcal{g}$ corresponding to $\mathrm{G}$. In particular,

$\left[{\underset{a}{a}}_{i}, v\right]=\frac{\nu^{2}}{N} \underline{b}_{i}$

The adjoint representation of $g$ reduces into a direct sum of $(2 j+1)$ dimensional irreducible representations of these $\mathrm{SU}(2)_{\omega}$ with multiplicities $c_{j}$, with $c_{1} \geqslant 1$.

The constant $N=\Sigma c_{j} j(j+1)(2 j+1) / 3$ is an interger $\geqslant 2$. It is a purely group theoretical constant which depends on the particular embedding of SU(2) Lie algebra in $\varrho($ for $g=\mathrm{SO}(3), N=2$, for $\mathcal{G}=\mathrm{SU}(3), N=3$ or 12 etc.)

Eq. (17e) has a simple interpretation if we use (14), since then it reduces to

$M_{\alpha \beta} a_{i}^{\beta}=c^{2} n a_{i}^{\alpha}$,

which shows that the gauge fields are eigenvectors of the mass-matrix with the same eigenvalues. Finally, the angular equation (17f) implies

$(f(\omega), f(\omega))=\kappa^{2}$,

where $k$ is a constant.
We now return to the main problem, which is to solve the angular eqs. (17). We shall concentrate on the line of argument as the details will be published elsewhere. The first step is to show from (17) that the functions $\underset{\sim}{u}$ and $\underset{\sim}{v}$ which make up the scalar $f$ in (10) are not independent, but satisfy the relationship

$\underset{\sim}{f}=-\underset{\sim}{u}=\underset{\sim}{v}$.

For this purpose we use the identity

$L_{i} L_{j}+\partial_{i} \partial_{j}=\left(\delta_{i j}-\hat{r}_{i} \hat{r}_{j}\right) L^{2}$

for functions on the sphere, to show from (11) that $\underset{\sim}{u}(\omega)$ is actually a potential for $a(\omega)$, that is

$\underset{\sim}{a}(\omega)=-\frac{2}{L(L+1)} L \underset{\sim}{u}(\omega)$.

Using $f=2 u \sim \sim \sim \sim$ and eqs. (26) and (21) we then see that ( $\widetilde{1} 7 \mathrm{f})$ can be written in the form

$\partial_{i \sim} \underset{\sim}{v}+2[\underset{\sim}{a}, \underset{\sim}{u}]=-\left[L(L+1)+\frac{v^{2}}{N}\right] \underline{\sim}_{i}$.

Taking the (non-covariant) divergence of this equation and using (26) again, we obtain

$\partial^{2} \underset{\sim}{v}-2 L(L+1) \underset{\sim}{v}=2\left[L(L+1)+\frac{\nu^{2}}{N}\right] \underset{\sim}{u}$,

but since the operator $\partial^{2}-2 L(L+1)$ is negative definite (except in the trivial casc $L=0$ ) and since from

(11) and (17) $\underset{\sim}{u}$ is a spherical harmonic of $\operatorname{order} L$,

(28) implies that $v$ is a spherical harmonic of order $L$. Using this information in (28) itself, we find that $\underset{\sim}{v}$ is a constant multiple of $\boldsymbol{u}$. On the other hand, from (13) eq. (22) holds also for $\tilde{\boldsymbol{J}} \cdot \underset{\boldsymbol{a}}{\boldsymbol{a}}$, and since from (11) $\boldsymbol{J} \cdot \underset{\sim}{\boldsymbol{a}}$ is just $2(\underset{\sim}{u}+\underset{\sim}{v})$ we then have

$M_{\alpha \beta}\left(u^{\beta}+v^{\beta}\right)=c^{2} n\left(u^{\alpha}+v^{\alpha}\right)$.

In contrast, since $f=2 \underset{\sim}{u}+\underset{\sim}{v}$ is in the little group of $\phi(\omega)$ from (9), we have

$M_{\alpha \beta}\left(2 u^{\beta}+v^{\beta}\right)=0$.

One sees at once that for non-zero $c^{2} n$ and $f$, and for $\underset{v}{v}$ a constant multiple of $u$, eqs. (29) and (30) are possible only if $u+v$ is zero, in which case we have (24) as required. We also pick up the following relationship for the constants

$\nu^{2}=N L(L+1) / 2$ and $\kappa^{2}=N L^{2}(L+1)^{2} / 8$.

From (25) and (30) we note that (17f) can be reduced 
to a self-coupling equation for $f$, namely,

$\partial_{i} f=\frac{2}{L(L+1)}\left[f, L_{i} f\right]$

and the rest of the analysis consists in using the selfcoupling eq. (32) to show that $f(\omega)$ is linear in $\hat{r}$. For this purpose we note that since from (25) and (30) the real vector $f^{\alpha}(\omega)$ is a spherical harmonic of order $L$, we can make the expansion

$f^{\alpha}(\omega)=\sum_{m} t_{m}^{\alpha} Y_{m}^{L}(\omega), \quad t_{m}^{\alpha^{*}}=(-1)^{m} t_{-m}^{\alpha}$

Expressing the third component of (32) explicitly in terms of the structure constants of $\mathrm{G}$, and inserting (33) we obtain, using standard spherical harmonic analyses,

$$
\begin{aligned}
& \frac{s-r}{L(L+1)} c_{\beta \gamma}^{\alpha} t_{r}^{\beta} t_{s}^{\gamma}=t_{m}^{\alpha} \frac{\sqrt{4 \pi}}{2 L+1} \\
& \times \sum_{j} \sqrt{2 j+1} \frac{\left(\begin{array}{lll}
j & L & L \\
\mu & r & s
\end{array}\right)}{\left(\begin{array}{lll}
j & L & L \\
0 & 0 & 0
\end{array}\right)} \int \mathrm{d} \omega Y_{\mu}^{j^{*}}(\omega) \partial_{3} Y_{m}^{L}(\omega) \text {. }
\end{aligned}
$$

Similarly, inserting (33) into (23), we obtain

$\sum_{\alpha} t_{r}^{\alpha} t_{s}^{\alpha}=\kappa^{2} \frac{4 \pi}{2 L+1}(-1)^{r} \delta_{r,-s}$

But now consider the operator

$P_{\alpha \beta}=\lambda \sum_{r}(-1)^{r} t_{r}^{\alpha} t_{-r}^{\beta}, \quad$ where $\quad \lambda=\frac{2 L+1}{\sqrt{4 \pi} \kappa^{2}}$.

One sees at once that $P_{\alpha \beta}$ is a $(2 L+1)$-dimensional projection operator with the vectors $t_{r}^{\alpha}$ as eigenvectors of unit eigenvalue. Hence from (34) (and the anti-symmetry of $c_{\beta \gamma}^{\alpha}$ in $\beta$ and $\gamma$ ) we have

$\left(\delta_{\alpha \alpha^{\prime}}-P_{\alpha \alpha^{\prime}}\right) c_{\beta^{\prime} \gamma^{\prime}}^{\alpha^{\prime}} P_{\beta^{\prime} \beta} P_{\gamma^{\prime} \gamma}=0$,

which shows that the $f^{\alpha}(\omega)$ span a Lie subalgebra of dimension $(2 L+1)$. Furthermore, since $\mathcal{F}$ is compact, and from $(11), f(\omega)=\underset{\sim}{v}(\omega)$ is a commutator, $\mathcal{F}$ is a semi-simple algebra.

Now, eq. (33) shows that the spatial rotation group $\mathrm{SO}(3)$ acts on the linear space $\mathcal{F}$ by its $(2 L+1)$-dimensional irreducible representation, and the integration in eq. (34) shows that the structure constants of $\mathcal{F}$ are invariant under spatial rotations. Thus the rotation group is a group of automorphisms of $\mathfrak{F}$. But for a semi-simple Lie group every continuous automorphism is inner. Therefore there exists an $\mathrm{SO}(3)$ subalgebra, SO(3) I of $\mathcal{F}$, which implements the infinitesimal spatial rotations and hence acts irreducibly on $\mathcal{F}$. But a non-trivial irreducible $\mathrm{SO}(3)_{\mathrm{I}}$ action on $\mathcal{F}$ is impossible unless $\mathscr{F}=\mathrm{SO}(3)_{\mathrm{T}}$. It follows that the space spanned by $f(\omega)$ is 3 -dimensional, and $L=1$.

If there are several non-conjugated $\mathrm{SO}(3)$ subalgebras of $\varrho$ the Higgs mechanism will in general choose among them. Eqs. (32), (24) and (26) yield, up to an arbitrary orthogonal choice of basis in $\mathrm{SO}(3)_{\mathrm{I}}$

$f^{\alpha}=-\kappa \hat{r}_{\alpha}, \quad a_{i}^{\alpha}=\kappa \epsilon_{\alpha i j} \hat{r}_{j}, \quad$ where $\quad \kappa=\sqrt{N / 2}$. (38)

Since we had absorbed the charge $e$ in the definition of $f(\omega)$ and $a(\omega)$, eq. (38) is exactly the 't HooftPoTyakov solution for the charge $e_{N}=e \sqrt{2 / N}$. This charge, which varies with the embedding in $\mathrm{G}$, is the natural charge for $S O(3)_{I}$, and the monopole strength has the minimum value of $4 \pi / e_{N}$.

Eq. (38) completely solves the angular equations for $a(\omega)$ and it remains only to consider the Higgs fields. From (7) and (38), we see that it must satisfy

$\left(L_{i}+t_{i}\right) \phi(\omega)=0$,

where the $t_{i}$ are the generators of $\mathrm{SO}(3)_{\mathrm{I}}$, normalized in the conventional way. Eqs. (39) and (17b) show that the $S O(3)_{I}$ representation of $\phi(\omega)$ is fixed to be $t(t+1)=l(l+1)$. For given $l=t$, it is easy to construct explicit solutions for $\phi(\omega)$ in terms of linear combinations of real and imaginary parts of appropriate spherical harmonics with real coefficients.

The above results show that the condition of separation of variables is so strong that it reduces the solution for general $\mathrm{G}$ to that for $\mathrm{G}=\mathrm{SO}(3)$, and that for $S O(3)$ the solution differs from the original 't HooftPolyakov ansatz only in that the Higgs field can belong to an arbitrary integral representation of $\mathrm{SO}(3)$ (and then only if the spin and isospin compensate as in $(39))^{\ddagger 3}$. Thus the 't Hooft-Polyakov solution for G $=\operatorname{SO}(3)$ is essentially the only solution with separated variables for arbitrary $G$.

It remains only to discuss the radial equations. Using (39) to determine the constants and replacing. $l(l+1)$ by its gauge-invariant counterpart $t(t+1)$, the radial eqs. (18), (19) are easily seen to reduce to

$\neq 3$ This generalizes to arbitrary $G$ and arbitrary $\Phi$ a result obtained independently for $\mathrm{SO}(3)$ by Cremmer et al. [5]. 
$r^{2} H^{\prime \prime}=H\left\{t(t+1) K^{2}+\mu^{2}(S-r)(\sigma S+r)\right\}, \quad H=e_{N} c S$,

$r^{2} K^{\prime \prime}=K\left\{\left(K^{2}-1\right)+\frac{t(t+1)}{2} H^{2}\right\}, \quad K=1-R$,

where the boundary conditions are $K(0)=1, H(0)=0$ and $K \rightarrow 0, H \rightarrow e_{N} c r, r \rightarrow \infty$. These equations are derivable from the Hamiltonian (4) which now takes the form

$$
\begin{aligned}
\mathcal{H} & =\frac{4 \pi}{e_{N}^{2}} \int \frac{\mathrm{d} r}{r^{2}}\left\{\left(r K^{\prime}\right)^{2}+\frac{1}{2}\left(r H^{\prime}-H\right)^{2}+\frac{1}{2}\left(1-K^{2}\right)^{2}\right. \\
& \left.+\frac{t(t+1)}{2} H^{2} K^{2}+\frac{\mu^{2}}{4} H^{2}\left[\sigma S^{2}+\frac{4}{3}(1-\sigma) r S-2 r^{2}\right]\right\} .
\end{aligned}
$$

Eqs. (40) reduce to the usual ones [6] for $t=1, \sigma$ $=1$ and although it is probable that they have regular finite energy solutions for other values of $t$ and $\sigma$, we do not know for sure at present whether they do or not. If they have, these solutions give a mass-formula $m(t)=\mathcal{H}$ on account of the $t$-dependence in (41), and since from (22) the kinetic mass of the gauge fields is $c^{2} t(t+1) / N$, the $t=1$ solution is probably the one with lowest mass. In conclusion we should perhaps mention that the above solutions can be extended to include dyons by making the ansatz $A_{\mathrm{o}}^{\alpha}=\hat{r}_{\alpha}(J(r) / r)$.

Full details of the calculations will be published elsewhere. We would like to thank Professors M.
Epstein and A. Goldhaber for many valuable discussions and suggestions.

Note added: One can prove the existence of solutions for the radial equations for all values of the "isospin" $t$, hy using arguments very similar to those of Tyupkin et al. [7]. As a matter of fact the soliton mass $m(t)$ is an increasing function of $t$ with a finite limit for $t \rightarrow \infty$. We are indebted to John Rawnley for this last result.

\section{References}

[1] E. Abers and B.W. Lee, Phys: Reports 9C (1973) 1.

[2] G. 't Hooft, Nucl. Phy sics B79 (1974) 276;

A. Polyakov, JETP Lett. 20 (1974) 194.

[3] E. Corrigan, D.B. Fairlie, J. Nuyts and D.I. Olive, CERN preprint TH2102 (1976);

E. Corrigan and D.I. Olive, CERN preprint TH2147 (1976);

A. Goldhaber and D. Wilkinson, SUNY preprint ITP-SB76-34 (1976);

F. Englert and P. Windy, Univ. Libre, Bruxelles preprint (1976);

A. Chakrabarti, Ann. Inst. Poincaré 13 (1975) 235;

R. Shankar, Phys. Rev. 14D (1976) 1107.

[4] E. Weinberg and A. Guth, Columbia Univ. preprint CO2271-77 (1976).

[5] E. Cremmer, F. Schaposnik and J. Scherk, Ecole Normale Superieure preprint (1976).

[6] M. Prasad and C. Sommerfield, Phys. Rev. Lett. 35 (1975) 760.

[7] Yu.S. Tyupkin, V.A. Fateev and A.S. Shvarts, Theor. Matem. Fisika 26 (1975) 397. 